\title{
Search Frictions, Competing Mechanisms and Optimal Market Segmentation
}

\author{
Xiaoming Cair \\ Pieter Gautier ${ }^{2}$ \\ Ronald Wolthoff
}

1 Tongji University, PR China,

2 Faculty of Economics and Business Administration, VU University Amsterdam, and Tinbergen Institute, the Netherlands;

3 University of Toronto, Canada. 
Tinbergen Institute is the graduate school and research institute in economics of Erasmus University Rotterdam, the University of Amsterdam and VU University Amsterdam.

More TI discussion papers can be downloaded at http://www.tinbergen.nl

Tinbergen Institute has two locations:

Tinbergen Institute Amsterdam

Gustav Mahlerplein 117

1082 MS Amsterdam

The Netherlands

Tel.: +31(0)20525 1600

Tinbergen Institute Rotterdam

Burg. Oudlaan 50

3062 PA Rotterdam

The Netherlands

Tel.: +31(0)10 4088900

Fax: +31(0)10 4089031 


\title{
Search Frictions, Competing Mechanisms and Optimal Market Segmentation*
}

\author{
Xiaoming Cai ${ }^{\dagger} \quad$ Pieter Gautier ${ }^{\ddagger} \quad$ Ronald Wolthoff ${ }^{\S}$
}

May 12, 2016

\begin{abstract}
In a market in which sellers compete for heterogeneous buyers by posting mechanisms, we analyze how the properties of the meeting technology affect the allocation of buyers to sellers. We show that a separate submarket for each type of buyer is the efficient outcome if and only if meetings are bilateral. In contrast, a single market with all agents is optimal if and only if the meeting technology satisfies a novel condition, which we call "joint concavity." Both outcomes can be decentralized by sellers posting auctions combined with a fee that is paid by (or to) all buyers with whom the seller meets. Finally, we compare joint concavity to two other properties of meeting technologies, invariance and non-rivalry, and explain the differences.
\end{abstract}

JEL classification: C78, D44, D83.

Keywords: search frictions, matching function, meeting technology, competing mechanisms, heterogeneity.

*The idea for this paper grew out of earlier fruitful collaboration with James Albrecht, Ben Lester, Ludo Visschers and Susan Vroman. We thank Philipp Kircher, Guido Menzio, Michael Peters, Gábor Virág, and various seminar and conference participants for valuable comments. Ronald Wolthoff gratefully acknowledges financial support from the Connaught Fund at the University of Toronto.

${ }^{\dagger}$ Tongji University. E-mail: xiaoming@tongji.edu.cn.

${ }_{\ddagger}^{\ddagger}$ Vrije Universiteit Amsterdam and Tinbergen Institute. E-mail: p.a.gautier@vu.nl.

$\S$ University of Toronto. E-mail: ronald.p.wolthoff@gmail.com. 


\section{Introduction}

Recent work by Eeckhout and Kircher (2010b) has demonstrated that in a market in which sellers compete for buyers with private valuations by posting mechanisms, the process that governs meetings between agents - i.e., the meeting technology - is crucially important for equilibrium outcomes, such as the choice of trading mechanism and the degree of market segmentation. For example, if buyers randomly select a seller among the ones that maximize their expected payoff and sellers are unconstrained in the number of buyers that they can meet (urn-ball meetings), then auctions are useful instruments to identify the buyer with the highest valuation. The efficient equilibrium in this case consists of a single market in which all sellers post auctions and all buyer types pool, as this maximally spreads high-type buyers across sellers. However, if sellers can only meet one buyer at a time (bilateral meetings), low-type buyers may crowd out high-type buyers. In that case, sellers can offer more value to high-valuation buyers by excluding low-valuation buyers. In equilibrium, sellers induce perfect separation of buyers into homogeneous submarkets by posting prices.

This insight is important because it reveals that easily observable equilibrium outcomes, like trading mechanisms and market segmentation, can be informative of a fundamental but less observable characteristic of a market, i.e. the degree of frictions in the meeting process. However, inference along these lines requires knowledge of whether there exist other meeting technologies that lead to the same outcomes. After all, bilateral or urn-ball meetings - although nearly universal in the search literature ${ }^{1}$ - are not necessarily the most adequate description of meeting technologies in real-life markets. ${ }^{2}$ In this paper, we therefore derive necessary and sufficient conditions on the meeting technology under which it is optimal to have (i) perfect separation, i.e. a separate market for each type of buyer, or (ii) perfect pooling, i.e. a single market with all agents, for all distributions of buyer types.

To do so, we analyze an environment based on Eeckhout and Kircher (2010b), in which a continuum of homogeneous sellers tries to trade with heterogeneous buyers, subject to the frictions generated by an arbitrary meeting technology. After describing this environment in detail in section 2, we start our analysis in section 3 by considering the trade-off of a social planner between the desire to spread high-type buyers as much as possible and the risk of them being crowded out by low-type buyers. Throughout, we extensively make use of a oneto-one transformation of the probability-generating function of a meeting technology. This transformation - the probability that a seller meets at least one buyer with an arbitrary label or characteristic - is developed in a companion paper Cai et al. (2016) and greatly simplifies the analysis.

\footnotetext{
${ }^{1}$ See below for an overview of the literature.

${ }^{2}$ For example, it might be more reasonable to suppose that a seller can meet and learn the type of multiple but not all buyers who try to match with him.
} 
Our first result concerns the optimality of perfect separation. We find that bilateral meetings are not only sufficient for this outcome, but also necessary. That is, if one moves away from bilateral meetings by allowing a seller to meet multiple buyers (potentially with arbitrary small probability), then there exist distributions of buyer valuations for which perfect separation is no longer efficient. Intuitively, separation does not exploit the efficiency gains that arise from sellers ranking multiple buyers: with homogenous submarkets, any meetings beyond the first are meaningless since they always yield the seller a clone of the buyer that he has already met.

Although the necessity of bilateral meetings for perfect separation is a new result in the literature, it is perhaps not very surprising. Most of our attention therefore goes out to the optimality of a single market. We show that this is the efficient outcome if and only if the meeting technology satisfies a novel condition which we call "joint concavity." Loosely speaking, this condition guarantees that social surplus can be increased by merging any two submarkets, irrespective of their composition. Joint concavity is satisfied by the urn-ball meeting technology, which explains why pooling is the efficient outcome in e.g. Peters and Severinov (1997), but we also describe a number of other meeting technologies that exhibit this property.

In the second half of section 3, we describe-based on Cai et al. (2016) - how both the separating and the pooling outcome can be decentralized by each seller posting a second-price auction, combined with a meeting fee to be paid by (or to) each buyer meeting him. ${ }^{3}$ Intuitively, in a large market, sellers take buyers' equilibrium payoffs as given, making sellers the residual claimant on any extra surplus that they create and providing them with an incentive to post efficient mechanisms. Auctions guarantee that the good is allocated efficiently, while the meeting fees price any positive or negative externalities in the meeting process, providing all agents with a payoff equal to their social contribution.

We conclude our analysis by comparing our findings to existing results in section 4 . In particular, we discuss how joint concavity relates to two other properties of meeting technologies described in the literature: (i) invariance as introduced by Lester et al. (2015), and (ii) nonrivalry as introduced by Eeckhout and Kircher (2010b). We show that invariance is a sufficient (but not a necessary) condition for joint concavity, while non-rivalry is a necessary (but not a sufficient) condition, and we explain why this is the case. Finally, the appendix contains all proofs, while additional results and discussion can be found in the online appendix.

Related Literature. Most of the search literature assumes that meetings are either bilateral (e.g. Moen, 1997; Guerrieri et al., 2010; Menzio and Shi, 2011) or take place according to an urn-ball meeting technology (e.g. Peters, 1997; Burdett et al., 2001; Shimer, 2005; Albrecht et al., 2014; Auster and Gottardi, 2016). Eeckhout and Kircher (2010b) were the first to consider

\footnotetext{
${ }^{3}$ As we explain in more detail below, this mechanism reduces to posted prices when meetings are bilateral.
} 
arbitrary meeting technologies. ${ }^{4}$ Relative to their paper, we contribute by identifying conditions for pooling or separating equilibria that are both necessary and sufficient.

Lester et al. (2015) use arbitrary meeting technologies in a model in which buyers' valuations are realized after they arrive at sellers. Their paper has therefore no implications for market segmentation and focusses instead on the question when meeting fees arise as part of the equilibrium mechanism. They show that this is the case if and only if the meeting technology is not invariant. We discuss the relation between joint concavity and invariance in section 4 .

Cai et al. (2016) make a methodological contribution by introducing the alternative representation of meeting technologies that we also use here. They show that this representation keeps the analysis of competing mechanisms with arbitrary meeting technologies tractable, even when heterogeneity is two-sided, but they do not characterize the optimal degree of market segmentation. ${ }^{5}$ Instead, they establish existence and efficiency of the market equilibrium - a result we employ in section 3.2 - and derive conditions on the meeting technology such that sellers' beliefs about the buyers that they will attract are uniquely determined by the market utility condition. ${ }^{6}$

Finally, Cai (2016) uses the alternative representation to extend the efficiency result of Hosios (1990) to an environment in which workers can meet multiple firms, e.g., through on-the-job search. However, he considers random search and wage bargaining, as in Gautier et al. (2010), and is therefore silent on market segmentation as well.

\section{Environment}

Agents and Preferences. A static economy is populated by a measure 1 of sellers, indexed by $j \in[0,1]$, and a measure $\Lambda>0$ of buyers. Both types of agents are risk-neutral. Each seller possesses a single unit of an indivisible good, for which each buyer has unit demand. ${ }^{7}$ All sellers have the same valuation for their good, which we normalize to zero. A buyer's valuation is an independent draw from a distribution $F(x)$ with $0 \leq x \leq 1 .^{8}$ We impose no additional structure on $F(x)$, although we will sometimes pretend that buyers have either a low valuation

\footnotetext{
${ }^{4}$ Alternatively, some papers (e.g. Fraja and Sákovics, 2001; Lester and Wolthoff, 2014; Wolthoff, 2015; Lester et al., 2016) propose specific (classes of) meeting technologies that are neither bilateral nor urn-ball.

${ }^{5}$ With two-sided heterogeneity, a single market never arises, as documented before by e.g. Shi (2001), Shi (2002), Shimer (2005), Eeckhout and Kircher (2010a) and Albrecht et al. (2014) for bilateral or urn-ball meetings.

${ }^{6}$ In this paper, we use the standard assumption that sellers expect the most favorable queue in case of a multiplicity. See section 3.2 for a detailed discussion.

${ }^{7}$ Although we analyze a goods market, it is straightforward to cast our model in a labor market setting in which homogeneous firms post menus of wages to attract workers who differ in their productivity, as in Shi (2006). All our results carry over to such an environment.

${ }^{8}$ The assumption that all buyers have a (weakly) higher valuation than the seller is standard as well as innocuous. Buyers with lower valuations would simply never trade.
} 
$x_{1}$ or a high valuation $x_{2}$ when describing the intuition behind our results. ${ }^{9}$ Buyers observe their valuation before making any decisions. An agent's payoff is the sum of (i) his monetary transfers and (ii) his valuation if he possesses the good at the end of the period (and zero otherwise).

Search. In order to attract buyers, each seller posts and commits to a direct mechanism (hereafter: "mechanism"). ${ }^{10}$ A mechanism specifies an extensive form game that determines for each buyer $i$ a probability of trade and an expected payment as a function of: (i) the total number $n$ of buyers that meet with the seller; (ii) the valuation $x_{i}$ that buyer $i$ reports; and (iii) the valuations $x_{-i}$ reported by the $n-1$ other buyers. ${ }^{11}$

All identical mechanisms are treated symmetrically by buyers and are therefore said to form a submarket. After observing all submarkets, each buyer chooses the one in which he wishes to attempt to match. ${ }^{12}$ As standard in the literature (see e.g. Shimer, 2005), we capture the anonymity of the large market by assuming that: i) sellers can condition their strategies on the actions of buyers but not on their identities, and ii) identical buyers must use identical mixed strategies in equilibrium. Consequently, agents' search decisions can be summarized by three endogenous variables for each submarket: the measure $s$ of sellers, the measure $b$ of buyers, and the distribution $G(x)$ of valuations among these buyers.

Meeting Technology. Within a submarket, meetings between buyers and sellers are governed by a frictional process, the meeting technology, which we model as in Eeckhout and Kircher (2010b). ${ }^{13}$ That is, the probability $P_{n}(\lambda)$ that a seller meets $n \in \mathbb{N}_{0}=\{0,1,2, \ldots\}$ buyers only depends on the queue length $\lambda=b / s$ in the submarket, is twice-continuously differentiable, and satisfies $\sum_{n=0}^{\infty} n P_{n}(\lambda) \leq \lambda$, since the number of meetings cannot exceed the number of buyers in the submarket. Further, we assume that the allocation of buyers to sellers within each submarket is independent of types. ${ }^{14}$ In other words, if a fraction $\mu / \lambda \in[0,1]$ of the buyers in the submarket are labeled "blue," then the probability for a seller to meet $i$ blue buyers and

\footnotetext{
${ }^{9}$ Neither proposition 1 nor proposition 2 below is driven by the requirement that they should hold for all $F(x)$, i.e. they remain the same under the weaker requirement that they should hold for all $F(x)$ with only two points of support.

${ }^{10}$ The assumption that the homogeneous side of the market posts mechanisms is in line with e.g. Peters and Severinov (1997), Shi (2006), Eeckhout and Kircher (2010b) and Albrecht et al. (2014). The nature of the equilibrium may change if the heterogeneous side of the market posts, as in e.g. Delacroix and Shi (2013) and Albrecht et al. (2016). See also Delacroix and Shi (2016) for a study of whether buyers or sellers should post.

${ }^{11}$ See the online appendix for a precise definition. In line with most of the literature (e.g. Peters, 1997; Eeckhout and Kircher, 2010b; Lester et al., 2015, 2016), we abstract from mechanisms that condition on other mechanisms in the market. Epstein and Peters (1999) and Peters (2001) provide a detailed discussion.

${ }^{12}$ The assumption that a buyer can meet only one seller per period is standard in the directed search literature. See Albrecht et al. (2006), Galenianos and Kircher (2009), Kircher (2009), Gautier and Holzner (2014) and Wolthoff (2015) for papers that relax this assumption.

${ }^{13}$ Our assumptions about the meeting technology are adopted from Eeckhout and Kircher (2010b) and shared by any model using either urn-ball or bilateral meetings.

${ }^{14}$ Of course, the equilibrium (or planner's) allocation of buyers to submarkets can depend on types.
} 
$n-i$ other buyers equals

$$
P_{n}(\lambda)\left(\begin{array}{c}
n \\
i
\end{array}\right)\left(\frac{\mu}{\lambda}\right)^{i}\left(1-\frac{\mu}{\lambda}\right)^{n-i}
$$

Alternative Representation. Cai et al. (2016) show that the analysis of arbitrary meeting technologies is often greatly simplified by using an alternative representation of $P_{n}(\lambda)$. This alternative representation is the probability $\phi(\mu, \lambda)$ that a seller with a queue $\mu$ of blue buyers and a queue $\lambda-\mu$ of other buyers meets at least one blue buyer. We follow this approach here. Given the assumption regarding type-independent allocation of buyers, $\phi(\mu, \lambda)$ equals

$$
\phi(\mu, \lambda)=1-\sum_{n=0}^{\infty} P_{n}(\lambda)\left(1-\frac{\mu}{\lambda}\right)^{n}
$$

To simplify notation, we will often omit the arguments of $\phi$ and use subscripts to indicate its partial derivatives. ${ }^{15}$

Examples of Meeting Technologies. For future reference, it will be useful to formally define a few examples of meeting technologies that satisfy all our assumptions.

1. Urn-Ball. First explored by Butters (1977) and Hall (1977), this technology specifies that - within a submarket - each buyer is randomly allocated to one of the sellers. As a result, the number of buyers that meet a particular seller follows a Poisson distribution with mean equal to the queue $\lambda$. That is, $P_{n}(\lambda)=e^{-\lambda} \frac{\lambda^{n}}{n !}$ and $\phi(\mu, \lambda)=1-e^{-\mu}$. ${ }^{16}$

2. Bilateral. With this technology, each seller meets at most one buyer, i.e. $P_{0}(\lambda)+P_{1}(\lambda)=1$ or $\phi(\mu, \lambda)=P_{1}(\lambda) \frac{\mu}{\lambda}$, with $P_{0}(\lambda)$ strictly convex. A potential micro-foundation consists of randomly pairing agents and keeping only pairs that consist of one buyer and one seller, yielding $P_{1}(\lambda)=\frac{\lambda}{1+\lambda} \cdot{ }^{17}$

3. Pairwise Urn-Ball. This technology, described by Lester et al. (2015), is a variation on the urn-ball technology. Buyers first form pairs, after which each pair is randomly assigned to a seller in the submarket. That is, $P_{n}(\lambda)=0$ for $n \in\{1,3,5, \ldots\}$ and $P_{n}(\lambda)=e^{-\lambda / 2} \frac{(\lambda / 2)^{n / 2}}{(n / 2) !}$ for $n \in\{0,2,4, \ldots\}$, which implies $\phi(\mu, \lambda)=1-e^{-\mu\left(1-\frac{1}{2} \frac{\mu}{\lambda}\right)}$.

4. Multi-Platform. This technology consists of two platforms or rounds. In the first round, all $b$ buyers and a fraction $0<\alpha<1$ of the $s$ sellers in a submarket attempt to meet

${ }^{15}$ See Cai et al. (2016) for a proof that the relation between $\phi(\mu, \lambda)$ and $P_{n}(\lambda)$ is one-to-one.

${ }^{16}$ To keep the exposition concise, we omit the (straightforward) derivation of $\phi(\mu, \lambda)$ for each example.

${ }^{17}$ This micro-foundation can be found in the money search literature (see e.g. Kiyotaki and Wright, 1993). Some papers in the labor search literature provide an alternative, consisting of an urn-ball process augmented with the constraint that each seller can only contact one random buyer among the ones that wish to meet him, such that $P_{1}(\lambda)=1-e^{-\lambda}$ (see e.g. Albrecht et al., 2006; Galenianos and Kircher, 2009; Gautier and Wolthoff, 2009; Gautier et al., 2016). 
according to the random-pairing bilateral technology described above. The $\frac{b}{b+\alpha s} b=\frac{\lambda}{\lambda+\alpha} b$ buyers who fail to meet a seller then participate in the second round, in which they meet the remaining $(1-\alpha) s$ sellers according to an urn-ball process. That is,

$$
P_{n}(\lambda)= \begin{cases}\alpha \frac{\alpha}{\lambda+\alpha}+(1-\alpha) e^{-\xi} & \text { if } n=0 \\ \alpha \frac{\lambda}{\lambda+\alpha}+(1-\alpha) \xi e^{-\xi} & \text { if } n=1 \\ (1-\alpha) \frac{\xi^{n} e^{-\xi}}{n !} & \text { if } n \in\{2,3, \ldots\},\end{cases}
$$

where $\xi=\frac{\lambda^{2}}{(1-\alpha)(\lambda+\alpha)}$ is the queue length in the second round. This yields $\phi(\mu, \lambda)=$ $\alpha \frac{\mu}{\lambda+\alpha}+(1-\alpha)\left(1-e^{-\frac{\lambda \mu}{(1-\alpha)(\lambda+\alpha)}}\right) \cdot{ }^{18}$

\section{Planner's Problem and Market Equilibrium}

We start by analyzing the problem of a planner whose objective is to maximize social surplus, subject to the frictions generated by the meeting technology. To keep the exposition as simple as possible, we initially assume that the planner knows buyers' valuations, allowing him to provide different types of buyers with different instructions. This naturally raises the question whether the planner's solution would be different without that knowledge. We establish that this is not case using the result of Cai et al. (2016) that - for any meeting technology - the solution can be decentralized with a particular incentive-comptabile mechanism, which provides a way for a planner who does not know buyers' valuations to implement the same solution.

\subsection{Planner's Problem}

The problem of a planner who knows buyers' valuations consists of two parts. First, the planner has to allocate buyers and sellers to submarkets, creating a queue length and a distribution of buyer types at each seller. Second, the planner has to specify how trade will take place after buyers arrive at sellers. We solve these stages in reverse order.

Trading Rule. Once a number of buyers $n \in \mathbb{N}_{1} \equiv\{1,2,3, \ldots\}$ has arrived at a seller, surplus is clearly maximized by allocating the good to the buyer with the highest valuation. Cai et al. (2016) show that the expected surplus generated by this trading rule can be written as the integral of $\phi$.

\footnotetext{
${ }^{18}$ While this technology may seem more involved than the other examples, the two-round structure actually resembles the meeting process in various real-life markets: (i) buyers who cannot find a product at the local bazaar may subsequently submit a bid at an online auction site; (ii) workers who have trouble finding a job are often put in touch with firms by a public employment agency; and (iii) singles who fail to meet someone in a bar may subscribe to a dating website. Admittedly, the analogy is not perfect because terms of trade may differ across platforms in reality, which is ruled out here by the definition of a submarket.
} 
Lemma 1 (Cai et al., 2016). The surplus created by a seller with a queue $\lambda$ of buyers whose types are distributed according to the distribution $G(x)$ equals

$$
S(\lambda, G)=\int_{0}^{1} \phi(\lambda(1-G(x)), \lambda) d x
$$

Allocation of Buyers. Now consider the allocation of buyers to sellers. For each seller $j \in[0,1]$, the planner chooses - with a slight abuse of notation - a queue length $\lambda(j)$ and a distribution of buyer types $G(j, x)$ to maximize total surplus $\int_{0}^{1} S(\lambda(j), G(j, x)) d j$. Of course, the planner cannot allocate more buyers of a certain type than are available. Formally, $\int_{0}^{1} \lambda(j) \nu(j, B) d j \leq \Lambda \nu_{F}(B)$ for any Borel-measurable set $B$, where $\nu_{F}$ is the measure associated with $F$ and $\nu(j, \cdot)$ is the measure associated with $G(j, \cdot)$.

Perfect Separation. We first establish that bilateral meetings are a necessary and sufficient condition for the optimality of perfect separation. Recall that bilateral meeting technologies are defined by the property that $P_{0}(\lambda)+P_{1}(\lambda)=1$ for any $\lambda$, with $P_{1}(\lambda)$ strictly concave.

Proposition 1. Bilateral meetings are a necessary and sufficient condition for the planner to create a separate submarket for each type of buyer under any type distribution $F(x)$.

Sufficiency of bilateral meetings for perfect separation is a well-known result in the literature: a separate submarket for each active buyer type avoids the high degree of crowding-out that arises if high-type and low-type buyers visit the same submarket and sellers meet one of both at random. ${ }^{19}$

Necessity is however - to the best of our knowledge - a new result. To understand the intuition, suppose that a seller can meet two or more buyers with positive probability. With perfect separation, any meetings beyond the first are irrelevant - as a seller will always meet a clone of the first buyer - and the gain in surplus relative to a bilateral technology is zero. Letting one high-type and one low-type buyer swap submarket, however, provides a way to increase surplus. After all, there is a positive probability that both these buyers meet sellers who meet other buyers as well. In that case, the buyers' joint contribution to surplus was 0 before the swap, but $x_{2}-x_{1}$ after the swap (generated by the high-type buyer; the low-type buyer still contributes 0 ). Of course, this argument is complete only if a buyer's probability to meet a seller is the same in both submarkets, or otherwise the change in surplus associated with changing the buyers' meeting probabilities has to be taken into account. Necessity therefore follows from distributions in which buyers' types are arbitrarily close. In contrast, if types are discretely different, a meeting technology may give rise to perfect separation even though it is not strictly bilateral (see Eeckhout and Kircher, 2010b, for a detailed discussion).

\footnotetext{
${ }^{19}$ A planner may of course keep the lowest types out of the market altogether if a marginal seller can generate more surplus in a different submarket (see e.g. Eeckhout and Kircher, 2010b).
} 
Perfect Pooling. To state our main result regarding the optimality of a single market, we define a novel property of meeting technologies, which we call "joint concavity."

Definition 1. A meeting technology exhibits joint concavity if and only if $\phi(\mu, \lambda)$ is concave in $(\mu, \lambda)$, i.e.

$$
\phi_{\mu \mu} \phi_{\lambda \lambda} \geq \phi_{\mu \lambda}^{2}
$$

for all $0 \leq \mu \leq \lambda<\infty .^{20}$

The following proposition then establishes that joint concavity is closely related to the optimality of a single market.

Proposition 2. Joint concavity is a necessary and sufficient condition for the planner to send all agents to the same market under any type distribution $F(x)$.

The intuition for this result is straightforward. ${ }^{21}$ Joint concavity is sufficient because it implies that by merging two submarkets, the probability $\phi(\lambda(1-G(x)), \lambda)$ of a seller meeting at least one buyer with a valuation higher than $x$ will increase for all $x$. Since the surplus created by a submarket is the integral of $\phi$ over $x$, pooling all agents into one submarket is optimal. In contrast, necessity follows from distributions that have two points of support, with one of both being zero, i.e. $x_{1}=0$ and $x_{2}>0$. For these distributions, only the number of meetings with high-type buyers matters for surplus and (2) reduces to $x_{2} \phi\left(\lambda\left(1-G\left(x_{1}\right), \lambda\right)\right.$. Hence, if joint concavity of $\phi$ fails, there exist measures of low-type and high-type buyers such that either partially or completely separating them into multiple submarkets is optimal. We provide a detailed discussion of joint concavity in section 4 , after considering decentralization first.

\subsection{Market Equilibrium}

Equilibrium Definition. Next, we define the market equilibrium. ${ }^{22}$ To do so, let $R(m, \lambda, G)$ denote the expected payoff of a seller who posts a mechanism $m$ and attracts a queue of buyers $(\lambda, G)$. Further, let $U(x, m, \lambda, G)$ denote the expected payoff of a buyer with valuation $x$ who visits this seller. Each seller aims to maximize his revenue $R$, but must take into account that his queue $(\lambda, G)$ is endogenously determined and depends on the mechanism that he posts. In line with the literature, we follow the market utility approach. That is, given a tuple $(m(j), \lambda(j), G(j, \cdot))$ for each seller $j \in[0,1]$, let $\bar{U}(x)$ denote the highest expected payoff that a buyer with valuation $x$ can obtain, i.e. $\bar{U}(x)=\max _{j \in[0,1]} U(x ; m(j), \lambda(j), G(j, \cdot))$. A seller posting a mechanism $m$ then expects a queue satisfying

$$
U(x, m, \lambda, G) \leq \bar{U}(x) \text {, with equality for each } x \text { in the support of } G .
$$

\footnotetext{
${ }^{20}$ Condition (3) is necessary and sufficient for concavity, since $\phi_{\mu \mu} \leq 0$ for all meeting technologies.

${ }^{21}$ Here the advantage of using $\phi$ becomes apparent; the equivalent condition in terms of $P_{n}$, which we derive in the online appendix, is far less simple and intuitive.

${ }^{22}$ We provide a brief discussion here. See the online appendix for additional details.
} 
For many meeting technologies, (4) uniquely determines the seller's queue. In case of multiplicity, we follow McAfee (1993), Eeckhout and Kircher (2010b) and Auster and Gottardi (2016) by assuming that sellers are optimistic and expect the solution that maximizes their revenue. ${ }^{23}$ Finally, sellers expect a queue that gives them a non-positive payoff if no solution to (4) exists. An equilibrium can then be defined as follows.

Definition 2. A directed search equilibrium is a mechanism $m(j)$ and a queue $(\lambda(j), G(j, \cdot))$ for each seller $j \in[0,1]$, and a market utility $\bar{U}(x)$ for each type of buyer $x$, such that ...

1. each $(m(j), \lambda(j), G(j, \cdot))$ maximizes $R(m, \lambda, G)$ subject to equation (4);

2. aggregating queues across sellers does not exceed the total measure of buyers of each type;

3. incentive compatibility is satisfied, so buyers report their valuations truthfully.

Decentralization. The following proposition, which follows from Cai et al. (2016), states the main result regarding the market equilibrium: for any meeting technology, the planner's solution can be decentralized by having each seller post a second-price auction combined with a meeting fee, to be paid by each buyer meeting the seller. ${ }^{24}$

Proposition 3 (Cai et al.,2016). For any meeting technology, the planner's solution $\{\lambda(j), G(j, x)\}$ can be decentralized as a directed search equilibrium in which seller $j \in[0,1]$ posts a second-price auction and a meeting fee equal to

$$
\tau(j)=-\frac{\int_{0}^{1} \phi_{\lambda}(\lambda(j)(1-G(j, x)), \lambda(j)) d x}{\phi_{\mu}(0, \lambda(j))} .
$$

The intuition for this result is similar to the intuition for efficiency in many other directed search models. Since sellers take buyers' equilibrium payoffs as given, they are the residual claimant on any surplus that they create. This provides them with an incentive to post mechanisms that decentralize the planner's solution, which requires efficiency along two margins: (i) the allocation of buyers to sellers, and (ii) the allocation of the good given a queue of buyers.

The second-price auction fulfills the second requirement and provides each buyer with a payoff equal to the extra surplus that he creates when he has the highest valuation. To satisfy the first requirement however, each buyer must receive an expected payoff exactly equal to his marginal contribution to social surplus, which includes the externality that he may impose during the meeting process (e.g. by preventing a buyer with a higher valuation from meeting the seller). Because this externality is type-independent, it can be priced by the meeting fee

\footnotetext{
${ }^{23}$ We discuss this issue in detail and derive conditions for uniqueness in Cai et al. (2016).

${ }^{24}$ The meeting fee can be negative, turning it into a subsidy paid to each buyer. The equilibrium is of course not unique. Because of risk neutrality, the seller could for example condition the meeting fee on the number of buyers that shows up. However, all equilibria give rise to the same expected payoffs. See Peters and Severinov (1997), Albrecht et al. (2014), Lester et al. (2015) for detailed discussions of efficiency in related models.
} 
(5), which equals (the negative of) the spillovers that a buyer imposes on other buyers (the numerator) conditional on the event that he meets a seller (the probability of which is given by the denominator).

Posted Prices versus Auctions. It is worth highlighting that the equilibrium mechanism nests two popular trading mechanisms as special cases. As we discuss in more detail in the next section, technologies that exhibit joint concavity give rise to meeting fees that are nonpositive. For a subset of those technologies, the equilibrium meeting fee is exactly zero, reducing the equilibrium mechanism to a standard auction. In contrast, when meetings are bilateral, the second-price auction does not generate any revenue and the meeting fees, which are then strictly positive, act as posted prices.

\section{Categorization of Meeting Technologies}

Bilateral meetings are well understood, but joint concavity is a novel condition and warrants discussion. To better understand this condition, we compare it in this section to two other properties of meeting technologies described in the literature, invariance and non-rivalry. We show that invariance is a sufficient (but not a necessary) condition, while non-rivalry is a necessary (but not a sufficient) condition. Figure 1 summarizes this discussion.

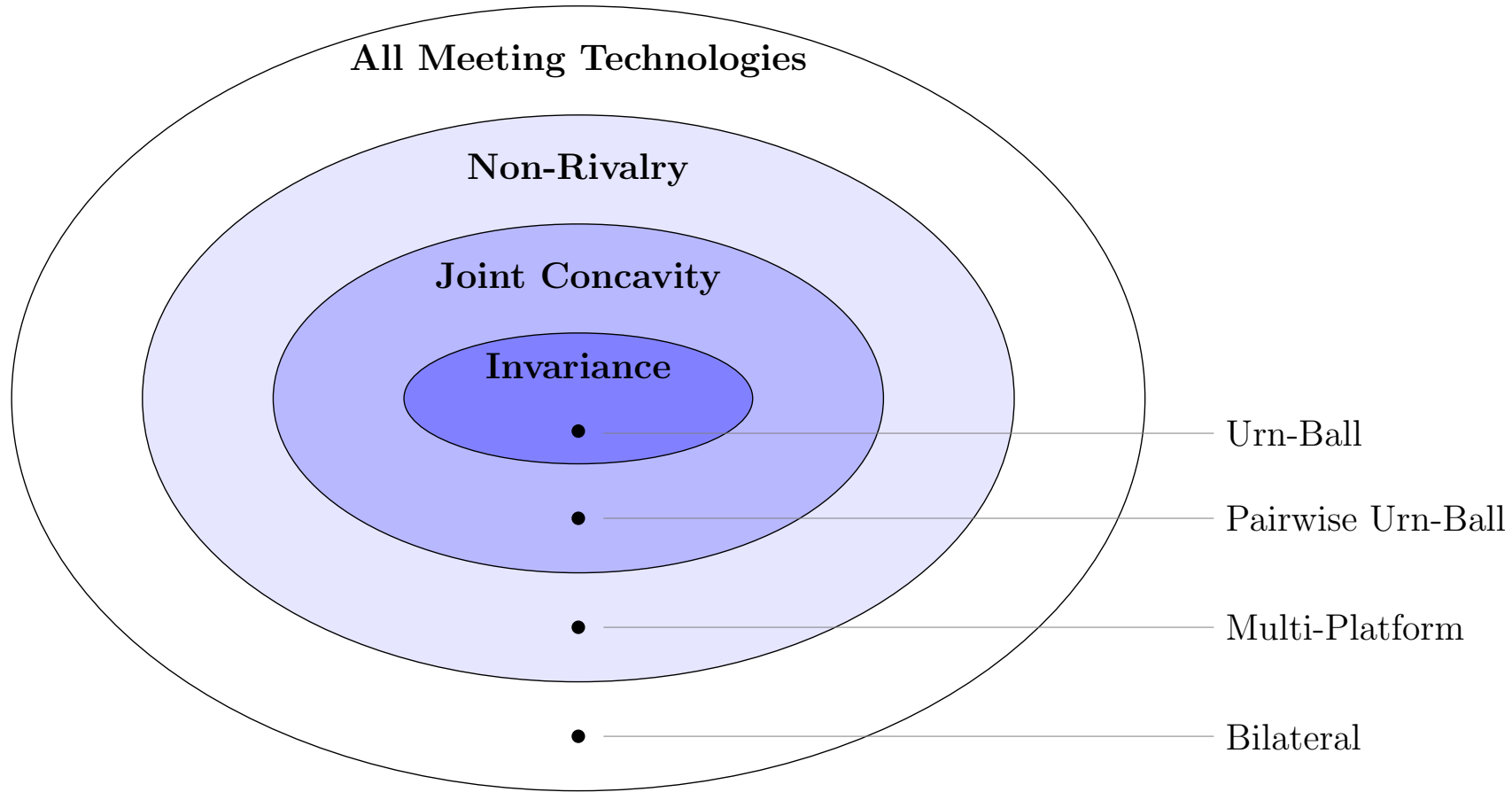

Figure 1: Venn Diagram of Meeting Technologies 
Invariance. Introduced by Lester et al. (2015), an invariant technology is one in which the queue of blue buyers $\mu$ at a seller is a sufficient statistic for the distribution of the number of meetings between blue buyers and that seller. Formally,

$$
\sum_{N=n}^{\infty} P_{N}(\lambda)\left(\begin{array}{l}
N \\
n
\end{array}\right)\left(\frac{\mu}{\lambda}\right)^{n}\left(1-\frac{\mu}{\lambda}\right)^{N-n}=P_{n}(\mu)
$$

for all $0 \leq \mu \leq \lambda<\infty$ and $n \in \mathbb{N}_{0}$. Perhaps the best-known example of an invariant technology is the urn-ball technology. ${ }^{25}$ In Lemma 2, we establish that if (6) holds for $n=0$, then it holds for all $n$. That is, invariance can alternatively be defined as the condition that the probability that a seller meets at least one of the $\mu$ blue buyers is independent of the number of other buyers visiting the same submarket.

Lemma 2. A meeting technology is invariant if and only if $\phi_{\lambda}(\mu, \lambda)=0$ for all $0 \leq \mu \leq \lambda<\infty$.

Using this lemma, it is easy to establish that invariance is a sufficient condition for joint concavity: if $\phi_{\lambda}=0$, then $\phi_{\mu \lambda}=\phi_{\lambda \lambda}=0$ and joint concavity is (weakly) satisfied. In words, invariance implies that meetings between blue buyers and sellers are unaffected by the presence of other buyers in the submarket. Joint concavity therefore reduces to concavity in the measure of blue buyers, which is always satisfied.

In contrast, invariance is not necessary for joint concavity; the condition for joint concavity can hold without $\phi_{\lambda}, \phi_{\mu \lambda}$ or $\phi_{\lambda \lambda}$ being zero. We prove this using the pairwise urn-ball technology: although this technology exhibits joint concavity, it is not invariant, as explained by Lester et al. (2015). Intuitively, when there are very few other buyers in the submarket, most buyer pairs consist of two blue types, making it likely that a seller will meet an even number of blue buyers. Adding additional other buyers to this submarket increases the probability that a buyer pair will consist of one blue and one other type, and that a seller will meet an odd number of blue buyers. This makes it more likely that a seller will meet at least one blue buyer, i.e. $\phi_{\lambda}>0$, violating invariance. ${ }^{26}$ The following proposition formalizes this.

Proposition 4. Invariance implies joint concavity, but joint concavity does not imply invariance.

Non-Rivalry. Eeckhout and Kircher (2010b) define a (purely) non-rival technology as one in which a buyer's probability to meet one of the sellers is not affected by the presence of other buyers in the market. We first establish that their definition is equivalent to $\phi_{\mu \lambda}(0, \lambda)=0$ for all $0 \leq \lambda<\infty$.

\footnotetext{
${ }^{25}$ Recall that urn-ball implies $\phi(\mu, \lambda)=1-e^{-\mu}$. As discussed in Lester et al. (2015), a second example of an invariant technology is the geometric distribution $P_{n}(\lambda)=\frac{\lambda^{n}}{(1+\lambda)^{n+1}}$, which yields $\phi(\mu, \lambda)=\frac{\mu}{1+\mu}$.

${ }^{26}$ We prove in the online appendix that $\phi_{\lambda} \geq 0$ is a necessary but not a sufficient condition for joint concavity.
} 
Lemma 3. A meeting technology is non-rival if and only if $\phi_{\mu \lambda}(0, \lambda)=0$ for all $0 \leq \lambda<\infty$.

To understand this expression, recall that $\phi(\mu, \lambda)$ represents the probability that a seller meets at least one blue buyer, which is clearly zero if $\mu=0$. The partial derivative $\phi_{\mu}(0, \lambda)$ captures how this changes if a single buyer (or more precisely, an arbitrarily small measure of buyers) in the queue becomes blue and must therefore equal the probability that this blue buyer succeeds in meeting the seller. Since meetings are type-independent, the same expression applies to all $\lambda$ buyers in the queue, irrespective of how many of them are blue. Non-rivalry then says that this meeting probability should be independent of $\lambda$.

It is easy to verify that the above examples of technologies that exhibit joint concavity, i.e. urn-ball and pairwise urn-ball, both satisfy non-rivalry. This is not a coincidence. As the following proposition establishes, all technologies that exhibit joint concavity are non-rival. However, not all non-rival technologies exhibit joint concavity.

Proposition 5. Joint concavity implies non-rivalry, but non-rivalry does not imply joint concavity.

To understand why non-rivalry is a necessary condition for joint concavity, consider a submarket with an arbitrarily small measure of blue buyers, i.e. $\mu \rightarrow 0$. A seller's probability of meeting at least one blue buyer in this submarket then goes to zero, $\phi(0, \lambda)=0$. Since this is the case irrespective of the queue $\lambda$ of other buyers, the derivatives of all orders of $\phi(0, \lambda)$ with respect to $\lambda$ are zero as well. By (3), joint concavity then requires $\phi_{\mu \lambda}(0, \lambda)=0$, which is exactly the condition for non-rivalry.

More generally, however, joint concavity requires that condition (3) is satisfied for any $0 \leq \mu \leq \lambda$ and not just for $\mu=0$. Hence, non-rivalry is not sufficient. The multi-platform technology is a good example. This technology is non-rival, because every buyer meets a seller with probability 1. However, this technology clearly does not satisfy joint concavity for sufficiently large $\alpha$, as it converges to a bilateral technology for $\alpha \rightarrow 1$. The proof of proposition 5 formalizes this and establishes that joint concavity is in fact violated for any $\alpha>0$. Hence, non-rivalry does not imply joint concavity. ${ }^{27}$

\section{Conclusion}

We study an environment in which sellers compete for heterogeneous buyers by posting mechanisms. Buyers can direct their search to the mechanism that maximizes their expected payoff, but may experience frictions in meeting a particular seller. We derive necessary and sufficient

\footnotetext{
${ }^{27}$ Joint with proposition 3, this result contradicts proposition 5 in Eeckhout and Kircher (2010b) which states that non-rivalry is a sufficient condition for a single market. The discrepancy originates in the fact that the proof of their proposition implicitly assumes invariance rather than non-rivalry when treating the trading probability for high-type buyers as independent of the queue of low-type buyers.
} 
conditions on the technology that governs these meetings under which either a separate submarket for each type of buyer or a single market with all agents are optimal. We find that perfect separation is the efficient equilibrium outcome if and only if meetings are bilateral, while perfect pooling is optimal if and only if the meeting technology satisfies a novel property, which we call "joint concavity."

\section{Appendix}

Proof of Lemma 1. This result first appeared in Cai et al. (2016). For completeness, we also provide a short proof here. The maximum valuation at a seller who meets $n \in \mathbb{N}_{1}$ buyers is an order statistic, distributed according to $G^{n}(x)$. Taking the expectation over $x$ and $n$, followed by integration by parts and using the Dominated Convergence Theorem to interchange summation and integration, yields

$$
S(\lambda, G)=\sum_{n=1}^{\infty} P_{n}(\lambda) \int_{0}^{1} x d G^{n}(x)=\int_{0}^{1}\left(1-\sum_{n=0}^{\infty} P_{n}(\lambda) G^{n}(x)\right) d x
$$

The result then follows because the rightmost integrand equals $\phi(\lambda(1-G(x)), \lambda)$.

Proof of Proposition 1. Part 1 (bilateral meetings imply perfect separation): Eeckhout and Kircher (2010b) demonstrate sufficiency for two types of buyers; we extend their result to arbitrary distributions. We do so in two steps: (i) buyers with different valuations must belong to different submarkets, and (ii) buyers with the same valuation must belong to the same submarket.

For (i), suppose that there exists a submarket with a measure $s$ of sellers and a queue $(\lambda, G)$. Because of lemma 1 and the fact that meetings are bilateral, the surplus created in this submarket equals

$$
s S(\lambda, G)=s P_{1}(\lambda) \int_{0}^{1}(1-G(x)) d x .
$$

Now suppose the planner would decompose this submarket into a separate submarket for each type of buyer $x$, allocating the $s$ sellers according to a distribution $H(x)$. Let $\widehat{\lambda}(x)=\lambda \frac{d G(x)}{d H(x)}$ denote the queue length in submarket $x$. A seller in this submarket then creates a surplus $P_{1}(\widehat{\lambda}(x)) x$, such that surplus across all submarkets equals

$$
s \int_{0}^{1} P_{1}(\widehat{\lambda}(x)) x d H(x)
$$

Clearly, if the planner chooses $H(x)=G(x)$, then $\widehat{\lambda}(x)=\lambda$ for all $x$ and surpluses $(7)$ and (8) are equal to each other. In that case, the marginal value of a seller in submarket $x$ equals 
$\left(P_{1}(\lambda)-\lambda P_{1}^{\prime}(\lambda)\right) x$. This value is increasing in $x$, which means that the allocation of sellers is suboptimal and surplus (8) can be increased by sending some sellers to different submarkets. Since $P_{1}(\lambda)$ is concave, $P_{1}(\lambda)-\lambda P_{1}^{\prime}(\lambda)$ is increasing in $\lambda$, and the planner can increase surplus by allocating relatively more sellers to the submarkets in which buyers have high valuations.

For (ii), suppose that there are two submarkets for buyers with valuation $x$. Let $s_{i}$ and $\lambda_{i}$ respectively denote the measure of sellers and the queue length in submarket $i \in\{1,2\}$. By the strict concavity of $P_{1}(\lambda)$, merging the two submarkets then increases surplus:

$$
x s_{1} P_{1}\left(\lambda_{1}\right)+x s_{2} P_{1}\left(\lambda_{2}\right)<x\left(s_{1}+s_{2}\right) P_{1}\left(\frac{s_{1} \lambda_{1}+s_{2} \lambda_{2}}{s_{1}+s_{2}}\right) .
$$

Part 2 (perfect separation implies bilateral meetings): We prove this result in two steps. We first prove that (i) if $P_{0}(\lambda)$ is not convex, then the planner may create multiple submarkets, even when all buyers are homogeneous, and (ii) if $P_{0}(\lambda)$ is convex and, for some $\Lambda>0, P_{0}(\Lambda)+$ $P_{1}(\Lambda)<1$, then there exists a two-type distribution of buyers such that perfect separation is not optimal. ${ }^{28}$

For (i), if $P_{0}(\lambda)$ is not convex, then by definition there exist $s, \lambda_{1}$, and $\lambda_{2}$ such that

$$
s P_{0}\left(\lambda_{1}\right)+(1-s) P_{0}\left(\lambda_{2}\right)<P_{0}\left(s \lambda_{1}+(1-s) \lambda_{2}\right) .
$$

Now, let the market be populated by a measure 1 of sellers and a measure $s \lambda_{1}+(1-s) \lambda_{2}$ of buyers with valuation 1 . A single market with all agents generates surplus $1-P_{0}\left(s \lambda_{1}+(1-s) \lambda_{2}\right)$. However, the surplus generated by two submarkets - one with $s$ sellers and $s \lambda_{1}$ buyers and the other one with the remaining buyers and sellers - equals $s\left(1-P_{0}\left(\lambda_{1}\right)\right)+(1-s)\left(1-P_{0}\left(\lambda_{2}\right)\right)$, which is higher because of (9).

For (ii), let the market be populated by a measure 1 of sellers, a measure $b_{1}$ of buyers with valuation $x_{1}$, and a measure $b_{2}$ of buyers with valuation $x_{2}$, satisfying $b_{1}+b_{2}=\Lambda$ and $x_{2}>x_{1}$. We will prove the claim by contradiction. Suppose the planner fully separates the two types of buyers and optimally allocates $s_{i}$ sellers to the submarket for valuation $x_{i}$, where $s_{1}+s_{2}=1$. Define queue lengths $\lambda_{i}=\frac{b_{i}}{s_{i}}$.

Let now a measure $\varepsilon$ of buyers with valuation $x_{1}$ and an equally large measure of buyers with valuation $x_{2}$ swap submarket, such that - in both submarkets - the queue lengths stay the same, but the composition of types becomes marginally more diverse. Again by lemma 1, social

\footnotetext{
${ }^{28}$ Of course, if $P_{0}(\Lambda)+P_{1}(\Lambda)<1$, then - by continuity - there exists a small neighborhood of $\Lambda$ for which $P_{0}+P_{1}<1$.
} 
surplus of this new allocation equals

$$
\begin{aligned}
\mathcal{S}(\varepsilon) & =s_{2}\left[\left(x_{2}-x_{1}\right) \phi\left(\frac{b_{2}-\varepsilon}{s_{2}}, \lambda_{2}\right)+x_{1} \phi\left(\lambda_{2}, \lambda_{2}\right)\right] \\
& +s_{1}\left[\left(x_{2}-x_{1}\right) \phi\left(\frac{\varepsilon}{s_{1}}, \lambda_{1}\right)+x_{1} \phi\left(\lambda_{1}, \lambda_{1}\right)\right] .
\end{aligned}
$$

Clearly, $\varepsilon=0$ corresponds to perfect separation. For this to be the optimal allocation, it must be the case that $\mathcal{S}(\varepsilon) \leq \mathcal{S}(0)$ for all $\varepsilon>0$. Hence, a necessary condition is that $\mathcal{S}^{\prime}(0) \leq 0$. Differentiating equation (10) and evaluating at $\varepsilon=0$ gives

$$
\mathcal{S}^{\prime}(0)=\left(x_{2}-x_{1}\right)\left(\phi_{\mu}\left(0, \lambda_{1}\right)-\phi_{\mu}\left(\lambda_{2}, \lambda_{2}\right)\right)
$$

For $x_{1} \rightarrow x_{2}$, the economy converges to the case in which all buyers are homogeneous. Because of the convexity of $P_{0}(\lambda)$, there will be a single market with all agents in this limit. That is, $\lambda_{1} \rightarrow \Lambda$ and $\lambda_{2} \rightarrow \Lambda$, such that equation (11) converges to

$$
\mathcal{S}^{\prime}(0)=\left(x_{2}-x_{1}\right)\left(\phi_{\mu}(0, \Lambda)-\phi_{\mu}(\Lambda, \Lambda)\right)
$$

Note that

$$
\phi_{\mu}(\mu, \lambda)=\sum_{n=1}^{\infty} \frac{n P_{n}(\lambda)}{\lambda}\left(1-\frac{\mu}{\lambda}\right)^{n-1}
$$

This implies that

$$
\frac{1}{x_{2}-x_{1}} \mathcal{S}^{\prime}(0)=\sum_{n=2}^{\infty} \frac{n P_{n}(\Lambda)}{\Lambda} \geq \sum_{n=2}^{\infty} \frac{2 P_{n}(\Lambda)}{\Lambda}=\frac{2\left(1-P_{0}(\Lambda)-P_{1}(\Lambda)\right)}{\Lambda}>0
$$

where the last strict inequality is by assumption. Hence, when $x_{1}$ is sufficiently close to $x_{2}$, perfect separation is not optimal and social surplus can be increased by slightly mixing the submarkets.

Proof of Proposition 2. Part 1 (joint concavity implies perfect pooling): To prove this result, suppose that there are two submarkets, indexed by $i \in\{1,2\}$, consisting of $s_{i}>0$ sellers who each have a queue $\left(\lambda_{i}, G_{i}\right)$. By lemma 1 , total surplus across the two submarkets is equal to

$$
s_{1} \int_{0}^{1} \phi\left(\lambda_{1}\left(1-G_{1}(x)\right), \lambda_{1}\right) d x+s_{2} \int_{0}^{1} \phi\left(\lambda_{2}\left(1-G_{2}(x)\right), \lambda_{2}\right) d x .
$$

We show a higher surplus can be generated by merging the two submarkets, creating one market with $s_{0}=s_{1}+s_{2}$ sellers, each with a queue $\lambda_{0}=\frac{s_{1} \lambda_{1}+s_{2} \lambda_{2}}{s_{1}+s_{2}}$ of buyers whose valuations 
are distributed according to

$$
G_{0}(x)=\frac{s_{1} \lambda_{1} G_{1}(x)+s_{2} \lambda_{2} G_{2}(x)}{s_{1} \lambda_{1}+s_{2} \lambda_{2}}
$$

Again by lemma 1, this combined market will create a surplus $s_{0} \int_{0}^{1} \phi\left(\lambda_{0}\left(1-G_{0}(x)\right), \lambda_{0}\right) d x$, which is larger than (13) because concavity of $\phi(\mu, \lambda)$ implies that

$$
s_{1} \phi\left(\mu_{1}, \lambda_{1}\right)+s_{2} \phi\left(\mu_{2}, \lambda_{2}\right) \leq s_{0} \phi\left(\frac{s_{1} \mu_{1}+s_{2} \mu_{2}}{s_{1}+s_{2}}, \frac{s_{1} \lambda_{1}+s_{2} \lambda_{2}}{s_{1}+s_{2}}\right) .
$$

Hence, a single market is optimal for technologies that exhibit joint concavity.

Part 2 (perfect pooling implies joint concavity): We prove this result by showing that if $\phi$ is not concave, there exists a two-type distribution of buyers such that one market is not optimal. Note that if $\phi$ is not concave, then - by the definition of concavity - there exist values $\alpha, \mu_{1}, \mu_{2}$, $\lambda_{1}$ and $\lambda_{2}$, such that

$$
\alpha \phi\left(\mu_{1}, \lambda_{1}\right)+(1-\alpha) \phi\left(\mu_{2}, \lambda_{2}\right)>\phi\left(\mu_{0}, \lambda_{0}\right)
$$

where $\mu_{0}=\alpha \mu_{1}+(1-\alpha) \mu_{2}$ and $\lambda_{0}=\alpha \lambda_{1}+(1-\alpha) \lambda_{2}$.

Consider now a market in which buyers' valuations are either $x_{1}$ or $x_{2}$, with $0<x_{1}<x_{2}$. Set the measure of high-type buyers equal to $\mu_{0}$ and the measure of low-type buyers equal to $\lambda_{0}-\mu_{0}$, while maintaining the assumption that the measure of sellers equals 1 . Then by Lemma 1 , the social surplus of creating a single market is $\mathcal{S}_{1}=\left(x_{2}-x_{1}\right) \phi\left(\mu_{0}, \lambda_{0}\right)+x_{1} \phi\left(\lambda_{0}, \lambda_{0}\right)$.

Now, decompose the single market into two submarkets $A$ and $B$, with seller measures $\alpha$ and $1-\alpha$, total queue lengths $\lambda_{1}$ and $\lambda_{2}$, and high-type queue lengths $\mu_{1}$ and $\mu_{2}$, respectively. ${ }^{29}$ The social surplus per seller for the two submarkets is

$$
\begin{aligned}
& S_{2}^{A}=\left(x_{2}-x_{1}\right) \phi\left(\mu_{1}, \lambda_{1}\right)+x_{1} \phi\left(\lambda_{1}, \lambda_{1}\right) \\
& S_{2}^{B}=\left(x_{2}-x_{1}\right) \phi\left(\mu_{2}, \lambda_{2}\right)+x_{1} \phi\left(\lambda_{2}, \lambda_{2}\right)
\end{aligned}
$$

and total surplus across the two submarkets equals $\mathcal{S}_{2}=\alpha S_{2}^{A}+(1-\alpha) S_{2}^{B}$.

In the limit $x_{1} \rightarrow 0$, the two submarkets create more surplus than the single market, i.e. $\mathcal{S}_{2}>\mathcal{S}_{1}$, if and only if

$$
x_{2}\left(\alpha \phi\left(\mu_{1}, \lambda_{1}\right)+(1-\alpha) \phi\left(\mu_{2}, \lambda_{2}\right)\right)>x_{2} \phi\left(\mu_{0}, \lambda_{0}\right)
$$

which holds because it is exactly equation (14). Hence, joint concavity is a necessary condition for a single market.

\footnotetext{
${ }^{29}$ This is possible because $\mu_{0}=\alpha \mu_{1}+(1-\alpha) \mu_{2}$ and $\lambda_{0}=\alpha \lambda_{1}+(1-\alpha) \lambda_{2}$.
} 
Proof of Proposition 3. This result follows from Cai et al. (2016). For completeness, we provide a short proof here, while referring to their paper for additional detail. The proof consists of two parts. First, we consider a seller who can choose the length and composition of his queue directly in a competitive market ("relaxed maximization problem"). By the first welfare theorem, the equilibrium in this market is Pareto optimal, which necessarily implies that it maximizes social net output as there is only one consumption good. Subsequently, we establish that a seller who posts the proposed equilibrium mechanism to attract an endogenously determined queue of buyers ("constrained maximization problem") implements the same solution.

Part 1 (relaxed maximization problem): For a given market utility function $\bar{U}(x)$, a seller chooses the queue $(\lambda, G)$ that maximizes his expected payoff, which equals the difference between surplus $S(\lambda, G)$ and the expected payoff that the seller has to offer to each of the buyers. That is,

$$
\int_{0}^{1} \phi(\lambda(1-G(z)), \lambda) d z-\int_{0}^{1} \bar{U}(z) d \lambda G(z) .
$$

Because the seller takes the market utility function as given, he is a residual claimant on any extra surplus that he creates. Hence, the seller will compare the marginal cost $\bar{U}(x)$ of attracting a buyer with valuation $x$ to this buyer's marginal contribution to surplus $T(x)$. To calculate $T(x)$, increase the measure of buyers with values around $x$, formally $[x, x+\Delta x]$, by $\varepsilon$ and denote the new market tightness and buyer value distribution as $\lambda^{\prime}$ and $G^{\prime}$ respectively. That is, $\lambda^{\prime}=\lambda+\varepsilon$, while $\lambda^{\prime}\left(1-G^{\prime}(z)\right)=\lambda(1-G(z))$ for $z>x$ and $\lambda^{\prime}\left(1-G^{\prime}(z)\right)=\lambda(1-G(z))+\varepsilon$ for $z<x$. By Lemma 1, the average contribution to surplus by buyers with values around $x$ is

$$
\begin{aligned}
\frac{S\left(\lambda^{\prime}, G^{\prime}\right)-S(\lambda, G)}{\varepsilon} & =\frac{1}{\varepsilon}\left(\int_{0}^{x} \phi(\lambda(1-G(x))+\varepsilon, \lambda+\varepsilon)-\phi(\lambda(1-G(x)), \lambda)\right) \\
& +\frac{1}{\varepsilon}\left(\int_{x}^{1} \phi(\lambda(1-G(x)), \lambda+\varepsilon)-\phi(\lambda(1-G(x)), \lambda)\right)
\end{aligned}
$$

Let $\varepsilon \rightarrow 0$, then the above equation converges to

$$
T(x)=\int_{0}^{1} \phi_{\lambda}(\lambda(1-G(z)), \lambda) d z+\int_{0}^{x} \phi_{\mu}(\lambda(1-G(z)), \lambda) d z .
$$

The solution to the relaxed maximization problem must therefore satisfy

$$
\bar{U}(x) \geq T(x) \text { for all } x, \text { with equality for all } x \in \operatorname{supp} G
$$

Part 2 (constrained maximization problem): Consider now a seller who posts a second-price auction and a meeting fee $\tau$, attracting a queue $(\lambda, G)$. A buyer with valuation $x$ in the support of $G$ meets the seller together with $n-1$ other buyers with probability $\frac{n P_{n}(\lambda)}{\lambda} \cdot{ }^{30}$ Hence, he

\footnotetext{
${ }^{30}$ See Eeckhout and Kircher (2010b) or Lester et al. (2015).
} 
pays the meeting fee $\tau$ with probability $\frac{1}{\lambda} \sum_{n=1}^{\infty} n P_{n}(\lambda)=\phi_{\mu}(0, \lambda)$ and trades with probability $\frac{1}{\lambda} \sum_{n=1}^{\infty} n P_{n}(\lambda) G(x)^{n-1}=\phi_{\mu}(\lambda(1-G(x)), \lambda)$. As a result, his expected payoff is

$$
U(x, \tau, \lambda, G)=-\phi_{\mu}(0, \lambda) \tau+\int_{0}^{x} \phi_{\mu}(\lambda(1-G(y)), \lambda) d y
$$

where the second term is the payoff from the auction, which-by standard results in auction theory - equals the integral over the trading probabilities (see e.g. Peters, 2013). A queue $(\lambda, G)$ is therefore compatible with an auction with fee $\tau$ if and only if

$$
\bar{U}(x) \geq U(x, \tau, \lambda, G) \text { for all } x, \text { with equality for all } x \in \operatorname{supp} G
$$

Clearly, if a queue $(\lambda, G)$ satisfies (16), then by setting the entry fee $\tau$ in equation (17) equal to

$$
\tau=-\frac{\int_{0}^{1} \phi_{\lambda}(\lambda(1-G(x)), \lambda) d x}{\phi_{\mu}(0, \lambda)}
$$

it also satisfies (18). Therefore, any queue chosen by an unconstrained seller who can buy queues directly at a price $\bar{U}(x)$ is also compatible with an auction with an entry fee.

Proof of Lemma 2. Part 1 (invariance implies $\phi_{\lambda}=0$ ): Evaluating the definition of invariance (6) in $n=0$ yields

$$
\sum_{N=0}^{\infty} P_{N}(\lambda)\left(1-\frac{\mu}{\lambda}\right)^{N}=P_{0}(\mu) .
$$

The left-hand side of this equation is $1-\phi(\mu, \lambda)$ and the right-hand side is independent of $\lambda$. Hence, $\phi_{\lambda}(\mu, \lambda)=0$ for all $0 \leq \mu \leq \lambda<\infty$.

Part $2\left(\phi_{\lambda}=0\right.$ implies invariance): Note that $\phi_{\lambda}(\mu, \lambda)=0$ for all $0 \leq \mu \leq \lambda<\infty$ implies that $\phi(\mu, \lambda)=\phi(\mu, \mu)$. By equation $(1), \phi(\mu, \mu)=1-P_{0}(\mu)$. Consequently, equation (19) must hold for all $0 \leq \mu \leq \lambda<\infty$. By standard results from analytic function theory (see e.g. Ahlfors, 1979, p.32), we can differentiate both sides of this equation $n$ times with respect to $\mu$, which yields

$$
\sum_{N=n}^{\infty} \frac{N !}{(N-n) !} P_{N}(\lambda)\left(-\frac{1}{\lambda}\right)^{n}\left(1-\frac{\mu}{\lambda}\right)^{N-n}=P_{0}^{(n)}(\mu),
$$

for all $0 \leq \mu \leq \lambda<\infty$. For $\mu=\lambda$, this gives $P_{0}^{(n)}(\mu)=\frac{n !}{(-\mu)^{n}} P_{n}(\mu)$. Substitute this into the right hand side of equation (20) and rearrange the term $\frac{n !}{(-\mu)^{n}}$ to the left hand side gives $(6)$. Hence, $\phi_{\lambda}=0$ implies invariance. 
Proof of Proposition 4. Part 1 (invariance implies joint concavity): This result follows immediately from lemma 2: $\phi_{\lambda}(\mu, \lambda)=0$ for all $0 \leq \mu \leq \lambda<\infty$ implies that $\phi_{\lambda \lambda}(\mu, \lambda)=$ $\phi_{\mu \lambda}(\mu, \lambda)=0$ for all $0 \leq \mu \leq \lambda<\infty$, which in turn implies that equation (3) is satisfied.

Part 2 (joint concavity does not imply invariance): Consider the pairwise urn-ball technology, which satisfies $\phi(\mu, \lambda)=1-e^{-\mu\left(1-\frac{1}{2} \frac{\mu}{\lambda}\right)}$. Since $1-e^{-y}$ is an increasing, concave function, a sufficient condition for $\phi(\mu, \lambda)$ to be concave is that the map $(\mu, \lambda) \rightarrow \mu\left(1-\frac{1}{2} \frac{\mu}{\lambda}\right)$ is concave. ${ }^{31}$ The Hessian of this map is indeed negative semi-definite. However, the technology is not invariant, as

$$
\phi_{\lambda}(\mu, \lambda)=\frac{1}{2} \frac{\mu^{2}}{\lambda^{2}} e^{-\mu\left(1-\frac{1}{2} \frac{\mu}{\lambda}\right)}>0 .
$$

Hence, joint concavity does not imply invariance.

Proof of Lemma 3. As shown by Lester et al. (2015), non-rivalry is satisfied if and only if $\frac{\partial}{\partial \lambda} \frac{1}{\lambda} \sum_{n=1}^{\infty} n P_{n}(\lambda)=0$, for all $0 \leq \lambda<\infty$. The desired result then follows directly from observing that $\phi_{\mu}(0, \lambda)=\frac{1}{\lambda} \sum_{n=1}^{\infty} n P_{n}(\lambda)$.

Proof of Proposition 5. Part 1 (joint concavity implies non-rivalry): For any meeting technology, $\phi(0, \lambda)=0$ for all $0 \leq \lambda<\infty$, i.e. the probability of meeting a blue buyer is zero if $\mu=0$, irrespective of the value of $\lambda$. This implies that $\frac{\partial^{n}}{\partial \lambda^{n}} \phi(0, \lambda)=0$ for all $n \in \mathbb{N}_{1}$.

Suppose now that a technology does not satisfy non-rivalry, i.e. $\phi_{\mu \lambda}(0, \lambda) \neq 0$. Then

$$
\phi_{\mu \mu}(0, \lambda) \phi_{\lambda \lambda}(0, \lambda)-\phi_{\mu \lambda}^{2}(0, \lambda)<\phi_{\mu \mu}(0, \lambda) \phi_{\lambda \lambda}(0, \lambda)=0
$$

i.e. $\phi$ does not exhibit joint concavity. Hence, joint concavity implies non-rivalry.

Part 2 (non-rivalry does not imply joint concavity): Consider the multi-platform technology. Starting from the expression for $\phi(\mu, \lambda)$ for this technology, one can derive

$$
\phi_{\mu \lambda}=-\left(1-e^{-\frac{\lambda \mu}{(1-\alpha)(\lambda+\alpha)}}-\frac{\lambda \mu}{(1-\alpha)(\lambda+\alpha)} e^{-\frac{\lambda \mu}{(1-\alpha)(\lambda+\alpha)}}\right) \frac{\alpha}{(\lambda+\alpha)^{2}} \leq 0
$$

which equals 0 (only) for $\mu=0$. Hence, the multi-platform technology is non-rival.

Further, we get

$$
\phi_{\lambda}=-\left(1-e^{-\frac{\lambda \mu}{(1-\alpha)(\lambda+\alpha)}}\right) \frac{\mu \alpha}{(\lambda+\alpha)^{2}}
$$

which is strictly negative for all $0<\mu \leq \lambda<\infty$ and $\alpha>0$. As we show in the online appendix, $\phi_{\lambda} \geq 0$ is a necessary condition for joint concavity. Hence, the multi-platform technology does not exhibit this property.

\footnotetext{
${ }^{31}$ See, for example, Theorem 5.1 in Rockafellar (1970, p.32).
} 


\section{References}

Ahlfors, L. (1979). Complex Analysis. McGraw-Hill.

Albrecht, J. W., Gautier, P. A., and Vroman, S. B. (2006). Equilibrium directed search with multiple applications. Review of Economic Studies, 73:869-891.

Albrecht, J. W., Gautier, P. A., and Vroman, S. B. (2014). Efficient entry with competing auctions. American Economic Review, 104(10):3288-3296.

Albrecht, J. W., Gautier, P. A., and Vroman, S. B. (2016). Directed search in the housing market. Review of Economic Dynamics, 19:218-231.

Auster, S. and Gottardi, P. (2016). Competing mechanisms in markets for lemons. mimeo.

Burdett, K., Shi, S., and Wright, R. (2001). Pricing and matching with frictions. Journal of Political Economy, 109:1060-1085.

Butters, G. (1977). Equilibrium distributions of sales and advertising prices. Review of Economic Studies, 44(3):465-491.

Cai, X. (2016). Entry efficiency and the meeting technology. mimeo.

Cai, X., Gautier, P. A., and Wolthoff, R. P. (2016). Meetings and mechanisms. mimeo.

Delacroix, A. and Shi, S. (2013). Pricing and signaling with frictions. Journal of Economic Theory, 148:1301-1332.

Delacroix, A. and Shi, S. (2016). Should buyers or sellers organize trade in a frictional market? mimeo.

Eeckhout, J. and Kircher, P. (2010a). Sorting and decentralized price competition. Econometrica, 78:539-574.

Eeckhout, J. and Kircher, P. (2010b). Sorting vs screening - search frictions and competing mechanisms. Journal of Economic Theory, 145:1354-1385.

Epstein, L. and Peters, M. (1999). A revelation principle for competing mechanisms. Journal of Economic Theory, 88(1):119-161.

Fraja, G. D. and Sákovics, J. (2001). Walras retrouvé: Decentralized trading mechanisms and the competitive price. Journal of Political Economy, 109(4):pp. 842-863.

Galenianos, M. and Kircher, P. (2009). Directed search with multiple job applications. Journal of Economic Theory, 114:445-471.

Gautier, P. A. and Holzner, C. (2014). Maximum matching in the labor market under incomplete information. mimeo.

Gautier, P. A., Moraga-Gonzalez, J. L., and Wolthoff, R. P. (2016). Search costs and efficiency: Do unemployed workers search enough? European Economic Review, 84:123-139. 
Gautier, P. A., Teulings, C. N., and Van Vuuren, A. (2010). On-the-job search, mismatch and efficiency. The Review of Economic Studies, 77(1):245-272.

Gautier, P. A. and Wolthoff, R. P. (2009). Simultaneous search with heterogeneous firms and ex post competition. Labour Economics, 16:311-319.

Guerrieri, V., Shimer, R., and Wright, R. (2010). Adverse selection in competitive search equilibrium. Econometrica, 78(6):1823-1862. mimeo.

Hall, R. (1977). Microeconomic Foundations of Macroeconomics, chapter An Aspect of the Economic Role of Unemployment. Macmillan, London.

Hosios, A. J. (1990). On the efficiency of matching and related models of search and unemployment. Review of Economic Studies, 57:279-298.

Kircher, P. (2009). Efficiency of simultaneous search. Journal of Political Economy, 117:861-913.

Kiyotaki, N. and Wright, R. (1993). A search-theoretic approach to monetary economics. American Economic Review, 83(1):63-77.

Lester, B., Visschers, L., and Wolthoff, R. (2015). Meeting technologies and optimal trading mechanisms in competitive search markets. Journal of Economic Theory, 155:1-15.

Lester, B., Visschers, L., and Wolthoff, R. P. (2016). Competing with asking prices. Theoretical Economics, forthcoming.

Lester, B. and Wolthoff, R. P. (2014). Interviews and the assignment of workers to jobs. mimeo.

McAfee, R. P. (1993). Mechanism design by competing sellers. Econometrica, 61(6):pp. 12811312 .

Menzio, G. and Shi, S. (2011). Efficient search on the job and the business cycle. Journal of Political Economy, 119:468-510.

Moen, E. R. (1997). Competitive search equilibrium. Journal of Political Economy, 105:385-411.

Peters, M. (1997). A competitive distribution of auctions. The Review of Economic Studies, 64(1):pp. 97-123.

Peters, M. (2001). Common agency and the revelation principle. Econometrica, 69(5):13491372.

Peters, M. (2013). The Handbook of Market Design, chapter Competing Mechanisms. Oxford University Press.

Peters, M. and Severinov, S. (1997). Competition among sellers who offer auctions instead of prices. Journal of Economic Theory, 75:141-179.

Rockafellar, R. T. (1970). Convex Analysis. Princeton University Press.

Shi, S. (2001). Frictional assignment I: Efficiency. Journal of Economic Theory, 98:232-260. 
Shi, S. (2002). A directed search model of inequality with heterogeneous skills and skill-biased technology. Review of Economic Studies, 69(2):467-491.

Shi, S. (2006). Wage differentials, discrimination and efficiency. European Economic Review, 50:849-875.

Shimer, R. (2005). The assignment of workers to jobs in an economy with coordination frictions. Journal of Political Economy, 113(5):996-1025.

Wolthoff, R. P. (2015). Applications and interviews: Firms' recruiting decisions in a frictional labor market. mimeo. 


\title{
Search Frictions, Competing Mechanisms and Optimal Market Segmentation
}

\author{
Online Appendix
}

\author{
Xiaoming Cai* ${ }^{*} \quad$ Pieter Gautier ${ }^{\dagger} \quad$ Ronald Wolthoff ${ }^{\dagger}$
}

May 3, 2016

\section{Joint Concavity Using $P_{n}(\lambda)$}

In the main text, we define joint concavity in terms of $\phi$, but an equivalent condition in terms of $P_{n}$, the actual primitive of the model, can be derived. ${ }^{1}$ Starting from the definition of $\phi$, taking partial derivatives yields

$$
\begin{aligned}
& \phi_{\mu \mu}=-\sum_{n=0}^{\infty}(n+2)(n+1) \frac{P_{n+2}}{\lambda^{2}}\left(1-\frac{\mu}{\lambda}\right)^{n}, \\
& \phi_{\mu \lambda}=\sum_{n=0}^{\infty}\left[(n+1) \frac{\lambda P_{n+1}^{\prime}-P_{n+1}}{\lambda^{2}}+(n+2)(n+1) P_{n+2} \frac{\mu}{\lambda^{3}}\right]\left(1-\frac{\mu}{\lambda}\right)^{n}, \\
& \phi_{\lambda \lambda}=-\sum_{n=0}^{\infty}\left[P_{n}^{\prime \prime}+2 \mu(n+1) \frac{\lambda P_{n+1}^{\prime}-P_{n+1}}{\lambda^{3}}+\frac{(n+2)(n+1) P_{n+2} \mu^{2}}{\lambda^{4}}\right]\left(1-\frac{\mu}{\lambda}\right)^{n} .
\end{aligned}
$$

Using the fact that $\sum_{n=0}^{\infty} a_{n} y^{n} \sum_{n=0}^{\infty} b_{n} y^{n}-\left(\sum_{n=0}^{\infty} c_{n} y^{n}\right)^{2}=\sum_{n=0}^{\infty} \sum_{i=0}^{n}\left(a_{i} b_{n-i}-c_{i} c_{n-i}\right) y^{n}$, the condition for joint concavity can then be written as

$$
\phi_{11} \phi_{22}-\phi_{12}^{2}=\sum_{n=0}^{\infty} Z_{n}\left(1-\frac{\mu}{\lambda}\right)^{n} \geq 0
$$

${ }^{*}$ Tongji University. E-mail: xiaoming@tongji.edu.cn.

$\dagger$ Vrije Universiteit Amsterdam and Tinbergen Institute. E-mail: p.a.gautier@vu.nl.

$\ddagger$ University of Toronto. E-mail: ronald.p.wolthoff@gmail.com.

${ }^{1}$ To save on notation, we suppress the argument of $P_{n}$ throughout this derivation. 
where, after some simplification, $Z_{n}$ equals

$$
Z_{n}=\sum_{i=0}^{n}\left[\frac{(i+2)(i+1) P_{i+2} P_{n-i}^{\prime \prime}}{\lambda^{2}}-(i+1)(n-i+1) \frac{\lambda P_{i+1}^{\prime}-P_{i+1}}{\lambda^{2}} \frac{\lambda P_{n-i+1}^{\prime}-P_{n-i+1}}{\lambda^{2}}\right] .
$$

\section{Mechanisms and Equilibrium Conditions}

Our description of the mechanism space and equilibrium conditions is similar to Eeckhout and Kircher (2010) and Auster and Gottardi (2016). See those papers for additional details.

Mechanism Space. A direct mechanism $m$ is an extensive form game, which determines buyers' trading probabilities and payoffs. To be precise, for a buyer with valuation $x$, meeting the seller joint with $n-1$ other buyers whose valuations are $\left(x_{1}, \ldots, x_{n-1}\right)$, the mechanism specifies

1. a trading probability $\theta_{n}\left(x ; x_{1}, \ldots, x_{n-1} ; m\right)$, symmetric in $x_{1}, \ldots, x_{n-1}$.

2. a transfer $t_{n}\left(x ; x_{1}, \ldots, x_{n-1} ; m\right)$, symmetric in $x_{1}, \ldots, x_{n-1}$.

Feasibility requires that the sum of the trading probabilities across all buyers meeting a seller does not exceed 1, i.e.

$$
\sum_{i=1}^{n} \theta_{n}\left(x_{i} ; x_{1}, \ldots, x_{i-1}, x_{i+1}, \ldots x_{n} ; m\right) \leq 1 \forall\left(x_{1}, \ldots, x_{n}\right) \in[0,1]^{n}
$$

Incentive Compatibility. Given a queue $(\lambda, G)$, the expected probability of trade for a buyer with valuation $x$ equals

$$
\theta(x ; m, \lambda, G)=\sum_{n=1}^{\infty} \frac{n P_{n}(\lambda)}{\lambda} \int \ldots \int \theta_{n}\left(x ; x_{1}, \ldots, x_{n-1} ; m\right) d G\left(x_{1}\right) \ldots d G\left(x_{n-1}\right)
$$

while the expected transfer equals

$$
t(x ; m, \lambda, G)=\sum_{n=1}^{\infty} \frac{n P_{n}(\lambda)}{\lambda} \int \ldots \int t_{n}\left(x ; x_{1}, \ldots, x_{n-1} ; m\right) d G\left(x_{1}\right) \ldots d G\left(x_{n-1}\right) .
$$

Incentive compatibility then specifies that buyers maximize their payoff by by truthfully reporting their type. That is,

$$
\theta(x ; m, \lambda, G) x_{i}-t(x ; m, \lambda, G)
$$

is maximized in $x=x_{i}$ for all $x_{i}$. 
Payoffs. If a mechanism $m$ is incentive compatible and attracts a queue $(\lambda, G)$, then the expected payoff of a buyer with valuation $x$ is

$$
U(x ; m, \lambda, G)=\theta(x ; m, \lambda, G) x-t(x ; m, \lambda, G)
$$

while the expected payoff of the seller is

$$
R(m, \lambda, G)=\lambda \int t(x ; m, \lambda, G) d G(x)
$$

Beliefs. Given a mechanism $m(j)$ and a queue $(\lambda(j), G(j, \cdot))$ for each seller $j \in[0,1]$, define the market utility $\bar{U}(x)$ as the highest payoff that a buyer with valuation $x$ can obtain, i.e.

$$
\bar{U}(x)=\max _{j \in[0,1]} U(x ; m(j), \lambda(j), G(j, \cdot)) .
$$

A queue $(\lambda, G)$ solves the market utility condition for a mechanism $m$ if

$$
U(x ; m, \lambda, G) \leq \bar{U}(x) \text { with equality for each } x \in \operatorname{supp} G
$$

Denote the set of queues $(\lambda, G)$ that solve (1) for a mechanism $m$ by $Q(m)$. A seller who posts a mechanism $m$ then expects to obtain a payoff

$$
\max _{\lambda, G}\{R(m, \lambda, G) \mid(\lambda, G) \in Q(m)\}
$$

where the max operator represents sellers' optimism in case $Q(m)$ contains more than one element. By convention, sellers expect a non-positive payoff if $Q(m)$ is empty. ${ }^{2}$

Aggregate Consistency. Consistency requires that aggregating queues across sellers does not exceed the total measure of buyers of each type. That is,

$$
\int_{0}^{1} \lambda(j) \nu(j, B) d j \leq \Lambda \nu_{F}(B)
$$

for any Borel-measurable set $B$, where $\nu_{F}$ is the measure associated with $F$ and $\nu(j, \cdot)$ is the measure associated with $G(j, \cdot)$.

\footnotetext{
${ }^{2}$ The motivation for this assumption is the following. A seller who offers all buyer types a payoff less than their market utility, irrespective of the queue, expects to attract no buyers, which yields a zero payoff. A seller who offers certain buyer types a payoff higher than their market utility, irrespective of the queue, expects to attract an infinite queue, which yields the seller a payoff that approaches minus infinity.
} 


\section{Invariance, Non-Rivalry and Pooling}

Invariance. Proposition 2 and 4 jointly imply that invariance is a sufficient condition for a pooling equilibrium, but not a necessary condition. The intuition for sufficiency is straightforward. Invariance implies that the presence of low-type buyers in a submarket has no effect on the meetings between high-type buyers and sellers. Surplus is therefore maximized by spreading high-type buyers evenly across all sellers, as opposed to concentrating them at a subset, to maximize the number of high-type buyers that will trade. A single market results.

To see why invariance is not necessary, consider again the pairwise urn-ball technology. As discussed in the main text, $\phi_{\lambda}>0$ for this technology. That is, adding low-type buyers to a submarket increases the probability that a seller will meet a high-type buyer. This feature violates invariance, but not joint concavity: the fact that the addition of low-type buyers to the submarket helps to spread the high-type buyers better across sellers strengthens the incentive to send all buyers to the same market. ${ }^{3}$

Non-Rivalry. Proposition 2 and 5 jointly imply that non-rivalry is a necessary condition for a pooling equilibrium, but not a sufficient condition. To understand why non-rivalry is necessary, consider a submarket with a single high-type buyer with valuation $x_{2}>0$ and a number of low-type buyers with valuation $x_{1} \rightarrow 0$, such that surplus only depends on the trading probability of the high-type buyer. Violation of non-rivalry would imply that this probability could be increased by sending either some low-type buyers (if $\phi_{\mu \lambda}(0, \lambda)<0$ ) or some sellers (if $\phi_{\mu \lambda}(0, \lambda)>0$ ) to a different submarket, contradicting the optimality of the single market associated with joint concavity.

To see why non-rivalry is not sufficient, consider again the multi-platform technology. As discussed in the main text, this technology is non-rival. However, the presence of low-type buyers in the submarket increases the chances for high-type buyers to be crowded out at one of the $\alpha s$ sellers in the first round, concentrating them at the $(1-\alpha) s$ second-round sellers in higher numbers than optimal. It is therefore better to send at least some low types to a separate submarket.

\section{$4 \quad$ Necessity and Insufficiency of $\phi_{\lambda} \geq 0$}

The fact that the pairwise urn-ball technology satisfies $\phi_{\lambda} \geq 0$ as well as joint concavity may raise the question how these two properties are related. The following proposition establishes that $\phi_{\lambda}(\mu, \lambda) \geq 0$ for all $0 \leq \mu \leq \lambda<\infty$ is a necessary but not a sufficient condition for joint

\footnotetext{
${ }^{3}$ This may raise the question how $\phi_{\lambda} \geq 0$ relates to joint concavity. We prove in section 4 of this online appendix that it is a necessary but not a sufficient condition.
} 
concavity.

Proposition 1. Joint concavity implies $\phi_{\lambda} \geq 0$, but $\phi_{\lambda} \geq 0$ does not imply joint concavity.

Proof. Part 1 (joint concavity implies $\phi_{\lambda} \geq 0$ ): We prove this result by contradiction. Suppose that there exists a meeting technology for which $\phi(\mu, \lambda)$ is concave in $(\mu, \lambda)$, but $\phi_{\lambda}\left(\mu_{0}, \lambda_{0}\right)<0$ in some point $\left(\mu_{0}, \lambda_{0}\right)$. Note that $\phi_{\mu \mu}<0$ for all technologies that exhibit joint concavity, hence $\phi(\mu, \lambda)$ must also be concave in $\lambda$ alone, i.e. $\phi_{\lambda \lambda} \leq 0$. In other words, $\phi_{\lambda}(\mu, \lambda)$ is a non-increasing function of $\lambda$, such that $\phi_{\lambda}\left(\mu_{0}, \lambda\right) \leq \phi_{\lambda}\left(\mu_{0}, \lambda_{0}\right)<0$ for all $\lambda>\lambda_{0}$. This implies that $\phi\left(\mu_{0}, \lambda\right) \leq \phi\left(\mu_{0}, \lambda_{0}\right)+\phi_{\lambda}\left(\mu_{0}, \lambda_{0}\right)\left(\lambda-\lambda_{0}\right)$ for all $\lambda>\lambda_{0}$. Let $\lambda \rightarrow \infty$ and thus $\phi_{\lambda}\left(\mu_{0}, \lambda_{0}\right)\left(\lambda-\lambda_{0}\right) \rightarrow-\infty$, such that $\phi\left(\mu_{0}, \lambda\right) \rightarrow-\infty$. Since $\phi$ is a probability, this leads to the required contradiction. Hence, concavity of $\phi(\mu, \lambda)$, i.e. joint concavity, implies $\phi_{\lambda} \geq 0$.

Part $2\left(\phi_{\lambda} \geq 0\right.$ does not imply joint concavity): Consider the following technology.

Minimum Demand. This technology consists of two rounds. In the first round, the $b$ buyers in the submarket are allocated to the $s$ sellers according to the urn-ball technology. In the second round, each seller draws a minimum demand requirement and operates only if the number of buyers that came to him weakly exceeds this minimum. ${ }^{4}$ We assume that the minimum demand requirements follow a geometric distribution, such that the minimum is weakly less than $n \in \mathbb{N}_{1}$ with probability $1-(1-\psi)^{n}$ for $0<\psi<1$. Hence, $P_{n}(\lambda)=e^{-\lambda} \frac{\lambda^{n}}{n !}\left(1-(1-\psi)^{n}\right)$ for $n \in \mathbb{N}_{1}$ and $P_{0}(\lambda)=1-\sum_{n=1}^{\infty} P_{n}(\lambda)=e^{-\psi \lambda}$.

This technology gives $\phi(\mu, \lambda)=1-e^{-\mu}-e^{-\psi \lambda}+e^{-\lambda \psi-\mu(1-\psi)}$. Hence, $\phi_{\lambda}=\psi e^{-\psi \lambda}\left(1-e^{-\mu(1-\psi)}\right)$, which is strictly positive. However, the determinant of the Hessian of $\phi$, evaluated in $\mu=0$, equals $-\psi^{2}(1-\psi)^{2} e^{-2 \lambda \psi}<0$, which means that $\phi$ is not concave. Hence, $\phi_{\lambda} \geq 0$ does not imply joint concavity.

\footnotetext{
${ }^{4}$ Geromichalos (2012) analyzes minimum demand requirements in a different context. Minimum class size requirements are also common in the matching between students and schools.
} 


\section{References}

Auster, S. and Gottardi, P. (2016). Competing mechanisms in markets for lemons. mimeo.

Eeckhout, J. and Kircher, P. (2010). Sorting vs screening - search frictions and competing mechanisms. Journal of Economic Theory, 145:1354-1385.

Geromichalos, A. (2012). Directed search and optimal production. Journal of Economic Theory, 147:2303-2331. 